\title{
Thue equations with composite fields
}

\author{
by \\ Yuri Bilu (Zürich) and Guillaume Hanrot (Talence)
}

1. Introduction. The history of numerical solution of Diophantine equations began in 1969, when Baker and Davenport [1] solved completely a system of two Pell equations. They used the well-known fact that every "large" solution gives rise to a "very small" value of a linear form $\Lambda\left(b_{1}, b_{2}\right)=\log \alpha_{0}+b_{1} \log \alpha_{1}+b_{2} \log \alpha_{2}$ (where $\alpha_{0}, \alpha_{1}$ and $\alpha_{2}$ are explicitly given algebraic numbers) at an integral point $\left(b_{1}, b_{2}\right)$. Using Baker's theory of logarithmic forms, they obtained a huge (around $10^{400}$ ) upper bound for $\max \left(\left|b_{1}\right|,\left|b_{2}\right|\right)$. After this, expanding $\log \alpha_{2} / \log \alpha_{1}$ into a continued fraction, they showed that $\left|\Lambda\left(b_{1}, b_{2}\right)\right|$ cannot be too small when $b_{1}$ and $b_{2}$ run through the integers below the huge bound. Therefore the system cannot have "large" solutions, while "small" solutions can be easily enumerated.

This idea was developed in various directions by Pethö, Tzanakis, de Weger, and many other authors. The subject became especially popular when Lenstra, Lenstra and Lovász [11] suggested a polynomially quick algorithm for finding an almost shortest vector in a lattice (referred to as LLL-algorithm in the sequel). The LLL-algorithm made it possible to extend the idea of Baker and Davenport to logarithmic forms in three or more variables, when continued fractions are not efficient any more. See [18, 12] for a detailed description of the methods, history of the subject and extensive bibliography up to 1989 .

In [4] we showed that one can solve Diophantine equations of Thue using only continued fractions (as Baker and Davenport did), and without involving the LLL-algorithm. This allowed us to solve completely Thue equations of rather high degree. In [5] we extended our method to superelliptic Diophantine equations (see also [3]).

1991 Mathematics Subject Classification: Primary 11Y50; Secondary 11B37, 11D41, 11D57, 11J86.

Research of the first author partially supported by SFB 343 "Diskrete Strukturen in der Mathematik", Bielefeld.

Research of the second author partially supported by CNRS GDR AMI. 
In this paper we show that the method of [4] becomes especially efficient if the number field related to the Thue equation contains a small subfield of degree at least 3 over $\mathbb{Q}$. We shall see that in this case one has to deal mainly with the subfield rather than with the whole field. We were motivated by the fact that such equations often occur in practice, for instance in the classical problem of primitive divisors $[17,20]$.

Using our method we managed to solve many totally real Thue equations of extremely high degree (up to 2505). See Section 7 for the details.

Acknowledgements. We are pleased to thank Attila Pethő and Benne de Weger for useful discussions and suggestions.

2. Notations. We consider the Thue equation

$$
F(x, y)=\mathcal{N}_{\mathbb{L} / \mathbb{Q}}(y-\alpha x)=a,
$$

where $a=a_{1} / a_{2}$ is a rational number, $\alpha$ an algebraic number of degree $n \geq 3$, and $\mathbb{L}=\mathbb{Q}(\alpha)$. We put

$$
f(y)=F(1, y)=\mathcal{N}_{\mathbb{L} / \mathbb{Q}}(y-\alpha) .
$$

We shall assume that the field $\mathbb{L}$ has a "small" subfield $\mathbb{K}$, of degree $m \geq 3$.

Let $\mathbb{K}$ have $s$ real and $2 t$ complex conjugate embeddings, where $s+2 t=$ $m$. We number the embeddings $\sigma_{1}, \ldots, \sigma_{m}$ so that $\sigma_{1}, \ldots, \sigma_{s}$ are the real embeddings, and

$$
\sigma_{s+i}=\left(\text { complex conjugation) } \circ \sigma_{s+i+t} \quad(1 \leq i \leq t) .\right.
$$

We write $\mathbb{K}_{i}=\sigma_{i}(\mathbb{K})$. We shall assume that $s \geq 1$; in particular, $\mathbb{K}$ has no roots of unity distinct from \pm 1 (in the case $s=0$ the equation is trivial; see [4, Section 2], for instance).

Put $l=n / m$ and fix an ordering $\alpha_{11}=\alpha, \alpha_{12}, \alpha_{13}, \ldots, \alpha_{m l}$ of the conjugates of $\alpha$ over $\mathbb{Q}$ so that for a fixed $i$ the numbers $\alpha_{i 1}, \ldots, \alpha_{i l}$ are conjugate over $\mathbb{K}_{i}$.

We use $O_{1}(\ldots)$ as a quantitative version of the usual $O(\ldots): A=O_{1}(B)$ means $|A| \leq B$.

For practical implementation of the method, one should be able to perform the following operations in the number field $\mathbb{K}$ :

(U) find a system of fundamental units;

(N) given a fractional ideal $I$ of the field $\mathbb{K}$, find a complete system of non-associate solutions of the norm equation

$$
N_{\mathbb{K} / \mathbb{Q}}(\beta)=a, \quad \beta \in I .
$$

(The units of $\mathbb{K}$ act on the solutions of (3) by multiplication. By a complete system of non-associate solutions of the equation (3) we mean any set 
of representatives of this action.) It is well known that any complete system of non-associate solutions is finite, and that problems $(\mathrm{U})$ and $(\mathrm{N})$ are effectively soluble [7, Ch. 2]. However, finding efficient algorithms for the practical resolution of these problems proved to be difficult, especially for fields of high degree. This is the main reason why the method is efficient only when the field $\mathbb{K}$ is "not very big". We do not discuss this problem, referring to $[8,15,14]$.

The purpose of the present paper is to show that the Thue equation (1) can be practically solved in reasonable time as soon as the problem (U) is solved and the problem $(\mathrm{N})$ is solved with $I=\mathcal{N}_{\mathbb{L} / \mathbb{K}}((1, \alpha))$.

Thus, fix once and for all a system $\eta_{1}, \ldots, \eta_{r}$ of basic units of the field $\mathbb{K}$, where $r=s+t-1$, and a complete system $\mathrm{M}$ of non-associate solutions of (3). In the important particular case when $|a|=1$ and $\alpha$ is an algebraic integer, we have $\mathrm{M}=\{1\}$.

Since $\mathbb{K}$ has no root of unity except \pm 1 , for any solution $\beta \in I$ of the equation (3) there exist $\mu \in \pm \mathrm{M}$ and $b_{1}, \ldots, b_{r} \in \mathbb{Z}$ such that $\beta=\mu \eta_{1}^{b_{1}} \ldots \eta_{r}^{b_{r}}$. Here $\pm \mathrm{M}=\{ \pm \mu: \mu \in \mathrm{M}\}$.

\section{General background}

3.1. The numbers $\varphi_{i}$. Fix a solution $(x, y) \in \mathbb{Z}^{2}$ of the equation (1).

Proposition 3.1.1. Put

$$
\begin{aligned}
& X_{0}=\left\{\begin{array}{c}
\left(\frac{2^{n-1}|a|}{\min _{\alpha_{i k} \notin \mathbb{R}}\left|f^{\prime}\left(\alpha_{i k}\right)\right| \cdot \min _{\alpha_{i k} \notin \mathbb{R}}\left|\operatorname{Im} \alpha_{i k}\right|}\right)^{1 / n} \\
\text { if } \mathbb{L} \text { is not totally real, } \\
\text { if } \mathbb{L} \text { is totally real, },
\end{array}\right. \\
& c_{1}=\frac{2^{n-1}|a|}{\min _{(i, k)}\left|f^{\prime}\left(\alpha_{i k}\right)\right|}, \quad c_{2}=\min _{(i, k) \neq\left(i^{\prime}, k^{\prime}\right)}\left|\alpha_{i k}-\alpha_{i^{\prime} k^{\prime}}\right|, \quad c_{3}=1.39 c_{1} c_{2}^{-1},
\end{aligned}
$$

(in the definition of $X_{0}$ both the minima run over the non-real conjugates of $\alpha)$. Let $(x, y)$ be an integer solution of (1).

(i) If $|x|>X_{0}$ then for some real conjugate $\alpha_{i_{0} k_{0}}$ we have

$$
\left|y / x-\alpha_{i_{0} k_{0}}\right| \leq c_{1}|x|^{-n} \text {. }
$$

(ii) If $|x|>X_{1}$ then

$$
y-\alpha_{i k} x=\left(\alpha_{i_{0} k_{0}}-\alpha_{i k}\right) x e^{O_{1}\left(c_{3}|x|^{-n}\right)} \quad\left((i, k) \neq\left(i_{0}, k_{0}\right)\right) .
$$

Proof. For (i) see [18, Lemma 1.1]. To prove (ii), write

$$
y-\alpha_{i k} x=\left(\alpha_{i_{0} k_{0}}-\alpha_{i k}\right) x\left(1+\frac{y / x-\alpha_{i_{0} k_{0}}}{\alpha_{i_{0} k_{0}}-\alpha_{i k}}\right) .
$$


Since $|x| \geq X_{1}$, we have

$$
\left|\frac{y / x-\alpha_{i_{0} k_{0}}}{\alpha_{i_{0} k_{0}}-\alpha_{i k}}\right| \leq \frac{1}{2} .
$$

But $1+z=e^{O_{1}(1.39|z|)}$ if the complex number $z$ satisfies $|z| \leq 1 / 2$ (see [18, p. 106]). Therefore (5) is a consequence of (6).

In concrete examples the constant $X_{1}$ is very small, and solutions satisfying $|x| \leq X_{1}$ can be easily enumerated. From now on, we assume that $|x|>X_{1}$, so that (4) and (5) hold for some $\left(i_{0}, k_{0}\right)$. Fix this $\left(i_{0}, k_{0}\right)$ and put

$$
\begin{aligned}
\varphi_{i}=\prod_{k=1}^{l}\left(y-\alpha_{i k} x\right) & (1 \leq i \leq m), \\
\psi_{i}=\prod_{k=1}^{l}\left(\alpha_{i_{0} k_{0}}-\alpha_{i k}\right) & \left(1 \leq i \leq m, i \neq i_{0}\right) .
\end{aligned}
$$

Then $\varphi:=\varphi_{1}=\mathcal{N}_{\mathbb{L} / \mathbb{K}}(y-\alpha x)$ and $\varphi_{i}=\sigma_{i}(\varphi)$. Also, immediately from (5) we deduce that

$$
\varphi_{i}=\psi_{i} x^{l} e^{O_{1}\left(c_{4}|x|^{-n}\right)} \quad\left(i \neq i_{0}\right),
$$

where $c_{4}=l c_{3}$. Since $\varphi_{1} \ldots \varphi_{m}=a$, we also obtain

$$
\varphi_{i_{0}}=\psi_{i_{0}} x^{(1-m) l} e^{O_{1}\left(c_{5}|x|^{-n}\right)}
$$

where $\psi_{i_{0}}=a\left(\prod_{i \neq i_{0}} \psi_{i}\right)^{-1}$ and $c_{5}=(m-1) c_{4}$. We unify (7) and (8) in

$$
\varphi_{i}=\psi_{i} x^{\varrho_{i}} e^{O_{1}\left(c_{5}|x|^{-n}\right)} \quad(1 \leq i \leq m),
$$

where

$$
\varrho_{i}= \begin{cases}l, & i \neq i_{0}, \\ (1-m) l, & i=i_{0} .\end{cases}
$$

We conclude this subsection with the following important property of the numbers $\varphi_{i}$.

Proposition 3.1.2. Among the $m-1$ numbers

$$
\varphi_{i} / \psi_{i} \quad\left(i \neq i_{0}\right)
$$

two at least are distinct.

Proof. Assume that the numbers (10) are all equal, and write this as

$$
P_{i}(\theta) / P_{i}\left(\alpha_{i_{0} k_{0}}\right)=P_{i^{\prime}}(\theta) / P_{i^{\prime}}\left(\alpha_{i_{0} k_{0}}\right) \quad\left(i, i^{\prime} \neq i_{0}\right),
$$

where $\theta=y / x$ and $P_{i}(T)=\prod_{k=1}^{l}\left(T-\alpha_{i k}\right)$. Note that the polynomials $P_{i}$ are pairwise distinct.

Let $\sigma_{i_{0} 1}, \ldots, \sigma_{i_{0} l}: \mathbb{L} \rightarrow \mathbb{C}$ be the extensions of $\sigma_{i_{0}}$ to $\mathbb{L}$ defined by $\sigma_{i_{0} k}(\alpha)=\alpha_{i_{0} k}$. Then for any $k \in\{1, \ldots, l\}$, the map $\tau_{k}:=\sigma_{i_{0} k} \sigma_{i_{0} k_{0}}^{-1}$ permutes the polynomials $P_{i}$, where $i \neq i_{0}$, and stabilizes $\theta$, a rational number. 
Hence, acting on (11) by $\tau_{1}, \ldots, \tau_{l}$, we obtain

$$
P_{i}(\theta) / P_{i}\left(\alpha_{i_{0} k}\right)=P_{i^{\prime}}(\theta) / P_{i^{\prime}}\left(\alpha_{i_{0} k}\right) \quad\left(i, i^{\prime} \neq i_{0}, 1 \leq k \leq l\right) .
$$

Fix distinct $i, i^{\prime} \in\{1, \ldots, m\} \backslash\left\{i_{0}\right\}$ (this is possible since $m \geq 3$ ). Then

$$
P_{i}(\theta) / P_{i^{\prime}}(\theta)=P_{i}\left(\alpha_{i_{0} k}\right) / P_{i^{\prime}}\left(\alpha_{i_{0} k}\right) \quad(1 \leq k \leq l) .
$$

Put $\phi=P_{i}(\theta) / P_{i^{\prime}}(\theta)$. Then the polynomial $P_{i}(T)-\phi P_{i^{\prime}}(T)$ has $l+1$ distinct roots $\theta, \alpha_{i_{0} 1}, \ldots, \alpha_{i_{0} l}$. Since its degree does not exceed $l$, it is identically zero. Since its leading coefficient is $1-\phi$, we have $\phi=1$. Thus, $P_{i}=P_{i^{\prime}}$, a contradiction. The proposition is proved.

3.2. The numbers $b_{i}$. Since $\mathcal{N}_{\mathbb{K} / \mathbb{Q}}(\varphi)=a$, we have

$$
\varphi=\mu \eta_{1}^{b_{1}} \ldots \eta_{r}^{b_{r}}
$$

where $\mu \in \pm \mathrm{M}$ and $b_{1}, \ldots, b_{r} \in \mathbb{Z}$. Put $\eta_{i j}=\sigma_{i}\left(\eta_{j}\right)$ and $\mu_{i}=\sigma_{i}(\mu)$, and let $A=\left[a_{i j}\right]_{1 \leq i, j \leq r}$ be the inverse of the matrix

$$
\left[\log \left|\eta_{i j}\right|\right]_{1 \leq i, j \leq r} .
$$

(The matrix (13) is non-singular, because its determinant is $\pm \min \left(1,2^{1-t}\right)$ times the regulator of the field $\mathbb{K}$.) Since

$$
\log \left|\varphi_{i}\right|=\log \left|\mu_{i}\right|+b_{1} \log \left|\eta_{i 1}\right|+\ldots+b_{r} \log \left|\eta_{i r}\right| \quad\left(i \neq i_{0}\right),
$$

it follows from (12) that

$$
b_{i}=\sum_{j=1}^{r} a_{i j} \log \left|\varphi_{j} / \mu_{j}\right|=\delta_{i} \log |x|+\lambda_{i}+O_{1}\left(c_{6}|x|^{-n}\right) \quad(1 \leq i \leq r),
$$

where

$$
\delta_{i}=\sum_{j=1}^{r} \varrho_{j} a_{i j}, \quad \lambda_{i}=\sum_{j=1}^{r} a_{i j} \log \left|\psi_{j} / \mu_{j}\right|, \quad c_{6}=c_{5} \sum_{j=1}^{r}\left|a_{i j}\right| .
$$

In particular, we obtain the following.

Proposition 3.2.1. If $|x| \geq X_{2}:=\max \left(X_{1},\left(2 \cdot 10^{10} c_{6}\right)^{1 / n}\right)$ then

$$
B:=\max \left(\left|b_{1}\right|, \ldots,\left|b_{r}\right|\right) \leq c_{7} \log |x|+c_{8},
$$

where

$$
c_{7}=\max _{1 \leq i \leq r}\left|\delta_{i}\right|, \quad c_{8}=\max _{1 \leq i \leq r}\left|\lambda_{i}\right|+10^{-10} .
$$

3.3. A large upper bound for $B$. In this subsection we obtain a huge upper bound for $B$ using Baker's theory. We apply a result of Baker and Wüstholz [2, p. 20], formulating it in a form convenient for the present paper.

TheOREM 3.3.1 (Baker-Wüstholz). Let $\beta_{0}, \ldots, \beta_{r}$ be complex algebraic numbers distinct from 0 and 1 , and $b_{1}, \ldots, b_{r+1}$ rational integers. Also, let

$$
d \geq\left[\mathbb{Q}\left(\beta_{0}, \ldots, \beta_{r}\right): \mathbb{Q}\right]
$$




$$
h_{i} \geq \max \left(h\left(\beta_{i}\right), d^{-1}\left|\log \beta_{i}\right|, d^{-1}\right) \quad(0 \leq i \leq r),
$$

where $h(. .$.$) is the absolute logarithmic height. Then either$

$$
\Lambda:=\log \beta_{0}+b_{1} \log \beta_{1}+\ldots+b_{r} \log \beta_{r}+b_{r+1} \pi i=0,
$$

or

$$
|\Lambda| \geq \exp \left(-c_{9} \log B^{\prime}\right) .
$$

Here $B^{\prime}=\max \left(\left|b_{1}\right|, \ldots,\left|b_{r}\right|,\left|b_{r+1}\right|, e\right)$, and

$$
c_{9}=18 \pi \cdot 32^{r+4}(r+3) !(r+2)^{r+3} d^{r+3} \log (2 d(r+2)) h_{0} \ldots h_{r} .
$$

REMARK 3.3.2. The parameters $n, h^{\prime}\left(\alpha_{1}\right), \ldots, h^{\prime}\left(\alpha_{n}\right), h^{\prime}(L)$ of the original theorem in [2] correspond to $r+2, h_{0}, \ldots, h_{r}, \pi / d, \log B^{\prime \prime}$ respectively in Theorem 3.3.1.

We have slightly modified the statement in [2], to allow inequalities in (18) and (19). It is often much easier (and quicker) to find an upper bound for the degree of a number field or for the height of an algebraic number, than to compute them exactly.

The following lemma is the case $h=1$ of [13, Lemma 2.2]:

LEMmA 3.3.3. Let $z$ and $C_{1}$ be positive numbers and $C_{2}$ an arbitrary real number. If $z \leq C_{1} \log z+C_{2}$ then $z \leq 2\left(C_{1} \log C_{1}+C_{2}\right)$.

By Proposition 3.1.2, there exist $i_{1}, i_{2} \in\{1, \ldots, m\} \backslash\left\{i_{0}\right\}$ such that

$$
\frac{\psi_{i_{2}} \varphi_{i_{1}}}{\psi_{i_{1}} \varphi_{i_{2}}} \neq 1 \text {. }
$$

On the other hand, as follows from (7),

$$
\frac{\psi_{i_{2}} \varphi_{i_{1}}}{\psi_{i_{1}} \varphi_{i_{2}}}=e^{O_{1}\left(2 c_{4}|x|^{-n}\right)} .
$$

Combining (22) and (23) with (12), we obtain

$$
1 \neq \beta_{0} \beta_{1}^{b_{1}} \ldots \beta_{r}^{b_{r}}=e^{O_{1}\left(2 c_{4}|x|^{-n}\right)},
$$

where

$$
\beta_{0}=\frac{\psi_{i_{2}}}{\psi_{i_{1}}} \cdot \frac{\sigma_{i_{1}}(\mu)}{\sigma_{i_{2}}(\mu)}, \quad \beta_{j}=\frac{\sigma_{i_{1}}\left(\eta_{j}\right)}{\sigma_{i_{2}}\left(\eta_{j}\right)} \quad(1 \leq j \leq r) .
$$

To compute the constant $c_{9}$ in this setting, we need to estimate $h\left(\beta_{0}\right), \ldots$, $h\left(\beta_{r}\right)$. This can be done using the well-known inequalities $h(a \pm b) \leq h(a)+$ $h(b)+\log 2$ and $h\left(a b^{ \pm 1}\right) \leq h(a)+h(b):$

$$
\begin{aligned}
& h\left(\beta_{0}\right) \leq 2 h(\mu)+2 l(2 h(\alpha)+\log 2), \\
& h\left(\beta_{j}\right) \leq 2 h\left(\eta_{j}\right) \quad(1 \leq j \leq r) .
\end{aligned}
$$


Denote by log the principal branch of the complex logarithm, that is, $-\pi<\operatorname{Im} \log z \leq \pi$. Then

$$
0<\left|\log \beta_{0}+b_{1} \log \beta_{1}+\ldots+b_{r} \log \beta_{r}+b_{r+1} \cdot \pi i\right| \leq 2 c_{4}|x|^{-n}
$$

for some $b_{r+1} \in \mathbb{Z}$. Comparing the imaginary parts, we obtain

$$
\left|b_{r+1}\right| \leq 1+\left|b_{1}\right|+\ldots+\left|b_{r}\right|+2 \pi^{-1} c_{4} X_{1}^{-n} \leq 1+0.45 l+B r
$$

because by the definition of $c_{4}$ and $X_{1}$ we have

$$
2 \pi^{-1} c_{4} X_{1}^{-n} \leq 2 \cdot 1.39 l(2 \pi)^{-1}<0.45 l .
$$

Therefore

$$
B^{\prime} \leq \max \left(e, c_{10}+c_{11} \log |x|\right),
$$

where $c_{10}=r c_{8}+1+0.45 l$ and $c_{11}=r c_{7}$.

As follows from $(25),(21)$ and $(27)$, either we have $B^{\prime}=e$, or

$$
\exp \left(c_{12}\left(c_{13}-B^{\prime}\right)\right) \geq \exp \left(-c_{9} \log B^{\prime}\right),
$$

where $c_{12}=n c_{11}^{-1}$ and $c_{13}=c_{10}+c_{12}^{-1} \log \left(2 c_{4}\right)$. Hence either we have $B^{\prime}=e$, or

$$
B^{\prime} \leq c_{12}^{-1} c_{9} \log B^{\prime}+c_{13} .
$$

In view of Lemma 3.3.3, this implies that

$$
B \leq B^{\prime} \leq B_{0}:=\max \left(e, 2\left(c_{12}^{-1} c_{9} \log \left(c_{12}^{-1} c_{9}\right)+c_{13}\right)\right) .
$$

4. Reduction of Baker's bound. In practice, the value of $B_{0}$ is too large for directly enumerating all possible $\left(b_{1}, \ldots, b_{r}\right)$. However, $B_{0}$ may be significantly reduced using continued fractions. As already mentioned in the introduction, a method of reduction was suggested by Baker and Davenport [1]. This method was developed by Tzanakis and de Weger [18], Pethö [12] and others. In this paper we use another modification of the Baker-Davenport method, suggested in [3-5].

The algorithm of reduction depends on whether $r=1$ or $r \geq 2$.

4.1. The case $r \geq 2$. Define $i_{1}$ by the condition

$$
\left|\delta_{i_{1}}\right|=\max _{1 \leq i \leq r}\left|\delta_{i}\right|=c_{7} .
$$

(Clearly, $\delta_{i_{1}} \neq 0$, because the matrix $A$ is non-singular.) Further, put

$$
\bar{\delta}_{i}=\delta_{i_{1}}^{-1} \delta_{i}, \quad \bar{\lambda}_{i}=\delta_{i_{1}}^{-1}\left(\delta_{i} \lambda_{i_{1}}-\delta_{i_{1}} \lambda_{i}\right) \quad(1 \leq i \leq r) .
$$

By the choice of $i_{1}$ we have $\left|\bar{\delta}_{i}\right| \leq 1$ for every $i$. Using (15), we obtain

$$
\begin{aligned}
b_{i} & =\delta_{i} \log |x|+\lambda_{i}+O_{1}\left(c_{6}|x|^{-n}\right) \\
& =\delta_{i} \delta_{i_{1}}^{-1}\left(b_{i-1}-\lambda_{i_{1}}+O_{1}\left(c_{6}|x|^{-n}\right)\right)+\lambda_{i}+O_{1}\left(c_{6}|x|^{-n}\right) \\
& =\bar{\delta}_{i} b_{i_{1}}-\bar{\lambda}_{i}+O_{1}\left(\left(1+\left|\bar{\delta}_{i}\right|\right) c_{6}|x|^{-n}\right)=\bar{\delta}_{i} b_{i_{1}}-\bar{\lambda}_{i}+O_{1}\left(2 c_{6}|x|^{-n}\right) .
\end{aligned}
$$


Fix $i_{2} \neq i_{1}$ and put $\bar{\delta}=\bar{\delta}_{i_{2}}$ and $\bar{\lambda}=\bar{\lambda}_{i_{2}}$. Then we can rewrite (29) as

$$
\left|b_{i_{2}}-\bar{\delta} b_{i_{1}}+\bar{\lambda}\right| \leq 2 c_{6}|x|^{-n} .
$$

Let $\kappa>2$ be a not very large number (at the end of this subsection we discuss the practical choice of $\kappa$ ). By the theorem of Dirichlet, there exists a positive integer $q \leq \kappa B_{0}$ such that

$$
\|q \bar{\delta}\| \leq\left(\kappa B_{0}\right)^{-1},
$$

where $\|\cdot\|$ is the distance to the nearest integer. In practice $q$ can be quickly found from the continued fraction expansion of $\bar{\delta}$. Multiplying (30) by $q$, we obtain

$$
\left\| \pm b_{i_{1}}\right\| q \bar{\delta}\|+q \bar{\lambda}\| \leq 2 c_{6} \kappa B_{0}|x|^{-n},
$$

where " \pm " should be "+" if $q \delta$ is smaller than the nearest integer and "_" otherwise.

It follows from (31) that $\left|b_{i_{1}}\right| \cdot\|q \bar{\delta}\| \leq \kappa^{-1}$. Therefore (32) implies that

$$
\|q \bar{\lambda}\|-\kappa^{-1} \leq 2 c_{6} \kappa B_{0}|x|^{-n} .
$$

If $\|q \bar{\lambda}\|>\kappa^{-1}$, which is heuristically plausible when $\kappa$ is large enough, then

$$
|x| \leq\left(\frac{2 c_{6} \kappa B_{0}}{\|q \bar{\lambda}\|-\kappa^{-1}}\right)^{1 / n} .
$$

Together with (17) this yields a new estimate for $B$ :

$$
B \leq c_{15}\left(\log B_{0}+\log \frac{c_{14} \kappa}{\|q \bar{\lambda}\|-\kappa^{-1}}\right),
$$

where $c_{14}=2 c_{6} e^{c_{8} / c_{15}}$ and $c_{15}=c_{7} / n$. In particular, when $\|q \bar{\lambda}\| \geq 2 \kappa^{-1}$, we have an estimate

$$
B \leq c_{15}\left(\log B_{0}+\log \left(c_{14} \kappa^{2}\right)\right)
$$

(compare this with the lemma from [1, Section 3]).

We took as a starting value $\kappa=10$, and tried the first reduction. If $\|q \lambda\|<2 \kappa^{-1}$, then we changed $\kappa$ to $10 \kappa$ and repeated the process.

The reduced bound for $B$ can be reduced again, using the same procedure, etc. Since in the case of a Thue equation the constant $c_{15}$ is usually rather small, the reduction is very efficient.

4.2. The case $r=1$. In this case the method of reduction is more or less the same as in the Tzanakis-de Weger paper [18]. We include some details for the sake of completeness.

Since $\mathbb{K}$ has a real embedding, we have $m=3$, and $\mathbb{K}$ has one real embedding $\sigma_{1}$ and a pair of complex conjugate embeddings $\sigma_{2}, \sigma_{3}$. We have $i_{0}=1$ and $\left\{i_{1}, i_{2}\right\}=\{2,3\}$; for instance, let it be $i_{1}=2$ and $i_{2}=3$. 
Now (24) can be rewritten as

$$
1 \neq \beta_{0} \beta_{1}^{b_{1}}=e^{O_{1}\left(2 c_{4}|x|^{-n}\right)}
$$

where

$$
\beta_{0}=\frac{\psi_{3}}{\psi_{2}} \cdot \frac{\sigma_{2}(\mu)}{\sigma_{3}(\mu)}, \quad \beta_{1}=\frac{\sigma_{2}\left(\eta_{1}\right)}{\sigma_{3}\left(\eta_{1}\right)} .
$$

Since $\sigma_{2}$ and $\sigma_{3}$ are complex conjugate, one has $\left|\beta_{0}\right|=\left|\beta_{1}\right|=1$. Also, $\beta_{1}$ is not a root of unity; otherwise, $\sigma_{2}\left(\eta_{1}^{N}\right)=\sigma_{3}\left(\eta_{1}^{N}\right)$ for some positive integer $N$. It would follow that $\eta_{1}^{N}$ is a Dirichlet unit of the field $\mathbb{Q}$, which means $\eta_{1}^{N}= \pm 1$, a contradiction.

Now rewrite (37) as

$$
0<\left\|\lambda+b_{1} \delta\right\| \leq 2 c_{4}|x|^{-n}
$$

with $\delta=\arg \beta_{1} /(2 \pi)$ and $\lambda=\arg \beta_{0} /(2 \pi)$, and continue as in the case $r \geq 2$.

4.3. Pathological reduction. In [4, Subsection 4.6] (see also [9]) we described various cases of "pathological" reduction: "semirational" and "totally rational" cases when $r \geq 2$, and multiplicative dependence of $\beta_{0}$ and $\beta_{1}$ when $r=1$. The method of reduction in the pathological cases is similar to that described above, and even more efficient. Since the "pathologies" occur in practice very seldom, we find it possible to omit their detailed analysis in this paper; if needed, it can be copied from [4] with insignificant changes.

5. Enumerating small $b_{i}$. Even when the upper bound for $B$ is reduced, enumerating all possible $\left(b_{1}, \ldots, b_{r}\right)$ can require extensive computations. One can imagine several ways to overcome this difficulty:

- using the continued fraction expansions of $\alpha$ (see $[12,18]$ for the details);

- sieving modulo several primes, as in $[19,16]$, for instance;

- using the Fincke-Pohst algorithm for finding all short vectors in a lattice, as in [21, 19], for instance.

We use a method suggested in [3], with some modifications introduced in $[5]$.

For $1 \leq i \leq r$ put $b_{i}^{\prime}=\bar{\delta}_{i} b_{i_{1}}-\bar{\lambda}_{i}$, where $i_{1}$ is defined from (28). Then $\left|b_{i}-b_{i}^{\prime}\right| \leq 2 c_{6}|x|^{-n}$. Since $X_{2} \geq\left(2 \cdot 10^{10} c_{6}\right)^{1 / n}$, we obtain

$$
\left|b_{i}-b_{i}^{\prime}\right|<10^{-10} \quad(1 \leq i \leq r)
$$

as soon as $|x|>X_{2}$. In particular,

$$
\left\|b_{i}^{\prime}\right\|<10^{-10} \quad(1 \leq i \leq r),
$$

and $b_{i}$ is the nearest integer to $b_{i}^{\prime}$.

Now we proceed as follows. Denote by $B_{0}^{\prime}$ the reduced bound for $B$. For every integer $b$ such that $|b| \leq B_{0}^{\prime}$, we put $b_{i_{1}}=b$, and compute the real 
numbers $b_{i}^{\prime}$ as above. Then for every $i$ we verify whether $\left\|b_{j}^{\prime}\right\|<10^{-10}$ or not. This condition trivially holds for $i=i_{1}$, but for $i \neq i_{1}$ it need not. If it is false for at least one $i$, then there is no solution $x$ with $|x|>X_{2}$ such that $b_{i_{1}}=b$, and we go to the next $b$.

The heuristic probability that the integer $b$ passes this severe test is $\left(2 \cdot 10^{-10}\right)^{r-1}$, quite a small number (when $r \geq 2$ ). For those very few $b$ that survive after the test, we use the second test, based on the following lemma. (We define $z^{1 / l}$ by $-\pi / l<\arg z^{1 / l} \leq \pi / l$.)

Lemma 5.1. For $i \neq i_{0}$ put $\omega_{i}=\left(\varphi_{i} / \psi_{i}\right)^{1 / l}$. Assume that $|x|>X_{3}:=$ $\max \left(X_{2},\left(1.3 \cdot 10^{10} c_{4}\right)^{1 /(n-1)}\right)$. If $l$ is odd then

$$
\left|x-\omega_{i}\right|<10^{-10} \text {. }
$$

If $l$ is even then we have either (40) or

$$
\left|x+\omega_{i}\right|<10^{-10} .
$$

Pr o of. We assume that $l$ is odd; the case of even $l$ is done similarly.

For $|z| \leq 1 / 2$ we have

$$
e^{z}=1+O_{1}\left(c_{16} z\right)
$$

where $c_{16}=2\left(e^{1 / 2}-1\right) \leq 1.3$. (This follows from the Schwarz lemma, applied to the function $e^{z}-1$ in the disc $|z| \leq 1 / 2$.) On the other hand,

$$
\omega_{i}=x e^{O_{1}\left(c_{4}|x|^{-n}\right)} \text {, }
$$

as follows from (7). Now (40) is an immediate consequence of (42) and (43). This proves the lemma.

The second test is performed as follows. Fix $i \neq i_{0}$ and compute $\varphi_{i}$ from (12), where $b_{i}$ are the nearest integers to $b_{i}^{\prime}$. Having $\varphi_{i}$, one can compute $\omega_{i}$ and check whether $\left\|\omega_{2}\right\| \leq 10^{-10}$ or not. If this fails then we go to the next $b$. Otherwise, we compute $x$ as the nearest integer to $\omega_{2}$ and check whether it corresponds to a solution $(x, y)$ of our equation $\left({ }^{1}\right)$. However, this option never happened in our computations.

REMARK 5.2. In the process of reduction one obtains an upper bound not only for $B$ but for $x$ as well, due to (34). Quite often, especially when $n$ is large, this bound does not exceed $X_{3}$ (or does exceed $X_{3}$ but is still reasonable). In this case enumerating small $b_{i}$ becomes superfluous.

6. The algorithm. Now we can summarize the contents of the previous sections in a formal algorithm. Before giving it, we notice that the numbers $\bar{\delta}_{i}$ depend only on $i_{0}$, and are independent of $\mu$ and $k_{0}$. Therefore the reduction and final enumeration can be performed simultaneously for all possible

$\left({ }^{1}\right)$ If $l$ is even then one has also to check the integer nearest to $-\omega_{i}$. 
pairs $\left(k_{0}, \mu\right)$, when $i_{0}$ is fixed. To do this, one must unify the constants depending on $k_{0}$ and $\mu$, by putting $\widehat{c}_{8}:=\max c_{8}\left(k_{0}, \mu\right)$, and redefining $c_{10}$ by substituting $\widehat{c}_{8}$ instead of $c_{8}$. (This would also affect the definitions of $c_{13}$, $c_{14}$, and $B_{0}$.)

At the starting point we are given the following data, which will be referred to as "the data":

- approximate values of $\alpha$ and all its conjugates;

- a system of fundamental units of the field $\mathbb{K}$ (for each unit approximate values of all its conjugates being required);

- the set $\mathrm{M}$ (again, for every $\mu \in \mathrm{M}$ we have to know approximate values of all its conjugates).

Here "approximate" means, depending on the situation, from fifty to one thousand decimal digits for both the real and imaginary part. If it turns out in course of solution that the precision is not sufficient, then the data should be recomputed with higher precision, and the algorithm re-executed from a suitable point (see Step 7).

Now we are in a position to describe the algorithm.

1. Compute matrix $A$, with highest possible precision.

2. Compute constants $c_{1}-c_{6}$ and $X_{1}-X_{3}$ with low precision (two decimal digits $\mathrm{OK}$ ).

3. Set $I_{0} \leftarrow 1$.

4. Set $i_{0} \leftarrow I_{0}$.

5. Compute the numbers $\bar{\delta}_{i}$, with highest possible precision, and the constants $c_{7}, \widehat{c}_{8}, c_{9}-c_{15}$, and $B_{0}$ with low precision.

6. For every pair $\left(k_{0}, \mu\right) \in\{1, \ldots, l\} \times \pm \mathrm{M}$ such that $\alpha_{i_{0} k_{0}} \in \mathbb{R}$ compute the corresponding set of $\bar{\lambda}_{i}$. If $\alpha_{i_{0} k_{0}} \notin \mathbb{R}$ for all $k_{0}$ then go to Step 9 .

7. Find a reduced bound for $B$, as described in Section 4 . If it turns out that the precision of $\bar{\delta}_{i}$ is not sufficient, then:

(a) recompute the data with a suitable precision;

(b) set $I_{0} \leftarrow i_{0}$;

(c) go to Step 4 .

8. Enumerate small $b_{i}$, as described in Section 5.

9. Set $i_{0} \leftarrow i_{0}+1$. If $i_{0} \leq s$ then go to Step 5 .

10 . For any $x \in \mathbb{Z}$ such that $|x| \leq X_{3}$ check whether $x$ corresponds to a solution of (1).

11. Collect all solutions obtained at Steps 8 and 10.

12. End. 
7. The real cyclotomic equation. As an example, we consider the real cyclotomic equation

$$
F_{P}(x, y):=\prod_{k=1}^{(P-1) / 2}\left(y-x \cdot 2 \cos \frac{2 \pi k}{P}\right)= \pm 1, \pm P,
$$

where $P>12$ is prime number. This equation occurs in the study of primitive divisors of Lucas and Lehmer numbers (see [17, 20]).

7.1. The field $\mathbb{K}$. Since the field $\mathbb{L}$ is abelian, for any $m$ dividing $(P-1) / 2$ there exists a subfield $\mathbb{K}$ of degree $m$. Thus, our method would be inefficient only if $n:=(P-1) / 2$ has no small divisors distinct from 1 and 2 , which happens quite seldom.

Thus, put

$$
m= \begin{cases}4 & \text { if } P \equiv 17(\bmod 24), \\ \text { the least odd prime divisor of } n & \text { otherwise. }\end{cases}
$$

Since the group $\operatorname{Gal}(\mathbb{L} / \mathbb{Q})$ is cyclic, there exists a single subfield $\mathbb{K}$ of $\mathbb{L}$ of degree $m$. The following lemma was used to compute a generator of $\mathbb{K}$ over $\mathbb{Q}$.

Lemma 7.1.1. Let a be a primitive root modulo $P$. Then the algebraic integer

$$
\xi_{0}=\sum_{k=0}^{n / m} 2 \cos \left(\frac{2 a^{m k} \pi}{P}\right)
$$

generates the field $\mathbb{K}$ over $\mathbb{Q}$. The conjugates of $\xi_{0}$ over $\mathbb{Q}$ are the numbers

$$
\xi_{i}=\sum_{k=0}^{n / m} 2 \cos \left(\frac{2 a^{m k+i} \pi}{P}\right) \quad(0 \leq i \leq m-1) .
$$

Pr o of. One verifies immediately that $\operatorname{Gal}(\mathbb{L} / \mathbb{K})$ stabilizes every $\xi_{i}$, and that $\operatorname{Gal}(\mathbb{K} / \mathbb{Q})$ acts on the set $\left\{\xi_{0}, \ldots, \xi_{m-1}\right\}$ transitively. This means that $\xi_{0}, \ldots, \xi_{m-1} \in \mathbb{K}$ and that $\xi_{i}$ are pairwise conjugate over $\mathbb{Q}$.

It remains to prove that $\xi_{0}$ generates $\mathbb{K}$. We shall use the following general observation.

Let $k \subseteq K \subseteq L$ be a tower of fields of characteristic zero, and assume that $\alpha \in L$ generates $L$ over $k$. Then the numbers

$$
\operatorname{Tr}_{L / K}\left(\alpha^{j}\right) \quad(1 \leq j \leq[L: K])
$$

generate $K$ over $k$.

(To prove this, notice that 
(i) the field $K$ is generated over $k$ by the coefficients of the minimal polynomial of $\alpha$ over $K$, and

(ii) these coefficients can be expressed as polynomials in the numbers (46) with integral coefficients.)

Now it is easy to complete the proof of the lemma. Since $a^{n}=a^{(P-1) / 2} \equiv$ $-1(\bmod P)$, one can rewrite $(45)$ as

$$
\xi_{i}=\sum_{k=0}^{(P-1) / m} \zeta^{a^{m k+i}} \quad(0 \leq i \leq m-1),
$$

where $\zeta$ is a primitive $P$ th root of unity. It follows immediately that $\operatorname{Tr}_{\mathbb{Q}(\zeta) / \mathbb{K}}\left(\zeta^{j}\right) \in\left\{\xi_{0}, \ldots, \xi_{m-1}\right\}$ for any $j \not \equiv 0(\bmod P)$. Hence $\mathbb{K}=$ $\mathbb{Q}\left(\xi_{0}, \ldots, \xi_{m-1}\right)$. Since $\operatorname{Gal}(\mathbb{K} / \mathbb{Q})$ is cyclic, at least one of the numbers $\xi_{i}$ generates $\mathbb{K}$. Since they are pairwise conjugate, any of them generates $\mathbb{K}$. The lemma is proved.

The set $M$ is $\{1\}$ when the right-hand side is \pm 1 , and consists of a single element when the right-hand side is $\pm P$ (because $P$ totally ramifies in $\mathbb{K}$ ).

7.2. Computing the constants, etc. The following lemmas show how to compute quickly $c_{1}$ and $c_{2}$.

Lemma $7.2 .1([9$, Lemme 3.6]). Let $l$ be an integer in $[1,(P-1) / 2]$, and

$$
\Psi(l)=\prod_{k=1, k \neq l}^{(P-1) / 2}|2 \cos (2 k \pi / P)-2 \cos (2 l \pi / P)| .
$$

Put

$$
p_{0}=\left\lfloor A \cos \left(\frac{\sqrt{3}}{3}\right) \frac{P}{\pi}\right\rfloor
$$

Then

$$
\min _{1 \leq l \leq(P-1) / 2}|\Psi(l)|=\min \left(\left|\Psi\left(p_{0}\right)\right|,\left|\Psi\left(p_{0}+1\right)\right|\right) .
$$

More precisely, this minimum is equal to $\left|\Psi\left(p_{0}\right)\right|$ if and only if

$$
\sin \left(2 p_{0} \pi / P\right) \sin \left(p_{0} \pi / P\right) \geq \sin \left(2\left(p_{0}+1\right) \pi / P\right) \sin \left(\left(p_{0}+1\right) \pi / P\right) .
$$

Since $\min \Psi(l)=\min _{i, k}\left|g^{\prime}\left(\alpha_{i k}\right)\right|$, this lemma allows one to compute $c_{1}$ very quickly.

Lemma $7.2 .2\left(\left[9\right.\right.$, Lemme 3.7]). We have $c_{2}=4 \sin (\pi / P) \sin (2 \pi / P)$.

All the constants are expressed in terms of the roots of $F(1, y)$ rather than its coefficients. Nevertheless, it is useful to have the following "closed" 
expression for $F(x, y)$, in particular, for enumerating the solutions with $|x| \leq$ $X_{3}$ (Step 10 of the algorithm).

Lemma 7.2.3 ([9, Lemme 3.8]). Let $\phi_{P}(x)=\left(x^{P}-1\right) /(x-1)$ be the Pth cyclotomic polynomial. Then

$$
F_{P}(x, y)=\left(\frac{2 x^{2}}{y+\sqrt{y^{2}-4 x^{2}}}\right)^{(P-1) / 2} \phi_{P}\left(\frac{y+\sqrt{y^{2}-4 x^{2}}}{2 x}\right) .
$$

7.3. Numerical results. The computations were done on a PC Pentium Pro $200 \mathrm{MHz}$, by a program written in C, using the PARI library version 1.915. We give in this table the value of the main constants for a few primes; the program, complete numerical details, and results for many other values of $P$ are available from the second author.

The last two columns of the following table contain respectively the total time of computation, and the time to compute and certify (using PARI) the fundamental units of $\mathbb{K}$ (both the times are in seconds). Compare the 4.3 seconds for the case $p=67$ with the 28 minutes of [4].

\begin{tabular}{c|c|c|c|c|c|c|c|c|c|c|c}
\hline$p$ & $m$ & $c_{6}$ & $c_{7}$ & $c_{8}$ & $c_{14}$ & $c_{15}$ & $B_{0}$ & $\begin{array}{c}B_{0} \\
\text { red }\end{array}$ & $X_{3}$ & Time & $\begin{array}{c}\text { Time } \\
(F U)\end{array}$ \\
\hline 67 & 3 & $8.01 \cdot 10^{12}$ & 4.65 & 2.00 & $1.18 \cdot 10^{14}$ & 0.141 & $2.05 \cdot 10^{28}$ & 7 & 7 & 4.3 & 1.0 \\
\hline 311 & 5 & $5.56 \cdot 10^{51}$ & 12.0 & 2.10 & $9.01 \cdot 10^{52}$ & 0.077 & $4.08 \cdot 10^{45}$ & 20 & 4 & 58.1 & 43.4 \\
\hline 977 & 4 & $4.73 \cdot 10^{153}$ & 76.2 & 7.89 & $2.50 \cdot 10^{157}$ & 0.156 & $2.58 \cdot 10^{42}$ & 80 & 2 & 60.5 & 16.5 \\
\hline 997 & 3 & $3.04 \cdot 10^{155}$ & 6.89 & 2.00 & $4.48 \cdot 10^{156}$ & 0.014 & $4.58 \cdot 10^{36}$ & 8 & 2 & 39.0 & 4.7 \\
\hline 5011 & 3 & $9.46 \cdot 10^{761}$ & 57.8 & 3.27 & $4.95 \cdot 10^{763}$ & 0.024 & $5.77 \cdot 10^{40}$ & 46 & 2 & 479.8 & 6.6 \\
\hline
\end{tabular}

We found that (for all $P$ above) the solutions of the equation $F_{P}(x, y)=$ \pm 1 are

$$
(0, \pm 1), \quad( \pm 1,0), \quad( \pm 1, \pm 1), \quad( \pm 1, \mp 1), \quad( \pm 1, \mp 2),
$$

and the solutions of the equation $F_{P}(x, y)= \pm P$ are $( \pm 1, \pm 2)$.

Combining this with [20, Lemma 1], we obtain the following result.

Corollary 7.3.1. The 311th, 977th, 997th, 5011th terms of any Lucas or Lehmer sequence have a primitive divisor.

In the forthcoming paper [6] (jointly with Paul Voutier) we show how the method of this paper, together with some ideas from [10], leads to the complete solution of the problem of primitive divisors. 


\section{References}

[1] A. Baker and H. Davenport, The equations $3 x^{2}-2=y^{2}$ and $8 x^{2}-7=z^{2}$, Quart. J. Math. Oxford Ser. (2) 20 (1969), 129-137.

[2] A. Baker and G. Wüstholz, Logarithmic forms and group varieties, J. Reine Angew. Math. 442 (1993), 19-62.

[3] Yu. Bilu, Solving superelliptic Diophantine equations by the method of GelfondBaker, preprint 94-09, Mathématiques Stochastiques, Univ. Bordeaux 2, 1994.

[4] Yu. Bilu and G. Hanrot, Solving Thue equations of high degree, J. Number Theory 60 (1996), 373-392.

[5] - - - Solving superelliptic Diophantine equations by Baker's method, Compositio Math. 112 (1998), 273-312.

[6] Yu. Bilu, G. Hanrot and P. M. Voutier, Existence of primitive divisors of Lucas and Lehmer numbers, submitted.

[7] Z. I. Borevich and I. R. Shafarevich, Number Theory, Academic Press, New York, 1966.

[8] H. Cohen, A Course in Computational Algebraic Number Theory, Grad. Texts in Math. 138, Springer, 1993.

[9] G. Hanrot, Résolution effective d'équations diophantiennes: algorithmes et applications, Thèse, Université Bordeaux 1, 1997.

[10] - Solving Thue equations without the full unit group, Math. Comp., to appear.

[11] A. K. Lenstra, H. W. Lenstra, jr., and L. Lovász, Factoring polynomials with rational coefficients, Math. Ann. 261 (1982), 515-534.

[12] A. Pethö, Computational methods for the resolution of Diophantine equations, in: R. A. Mollin (ed.), Number Theory: Proc. First Conf. Canad. Number Theory Assoc. (Banff, 1988), de Gruyter, 1990, 477-492.

[13] A. Pethő and B. M. M. de Weger, Products of prime powers in binary recurrence sequences, Part I: The hyperbolic case, with an application to the generalized Ramanujan-Nagell equation, Math. Comp. 47 (1987), 713-727.

[14] M. E. Pohst, Computational Algebraic Number Theory, DMV Sem. 21, Birkhäuser, Basel, 1993.

[15] M. E. Pohst and H. Zassenhaus, Algorithmic Algebraic Number Theory, Cambridge Univ. Press, 1989.

[16] N. Smart, The solution of triangularly connected decomposable form equations, Math. Comp. 64 (1995), 819-840.

[17] C. Stewart, Primitive divisors of Lucas and Lehmer numbers, in: Transcendence Theory: Advances and Applications, A. Baker and D. W. Masser (eds.), Academic Press, 1977.

[18] N. Tzanakis and B. M. M. de Weger, On the practical solution of the Thue equation, J. Number Theory 31 (1989), 99-132.

[19] - - - How to explicitly solve a Thue-Mahler equation, Compositio Math. 84 (1992), 223-288.

[20] P. Voutier, Primitive divisors of Lucas and Lehmer sequences, Math. Comp. 64 (1995), 869-888. 
[21] B. M. M. de Weger, Solving exponential diophantine equations using lattice basis reduction algorithms, J. Number Theory 26 (1987), 325-367.

Forschungsinstitut für Mathematik Algorithmique Arithmétique Expérimentale (A2X) ETH-Zentrum

CH-8092 Zürich, Switzerland

Current address:

Mathematisches Institut

Universität Basel

Rheinsprung 21

4051 Basel, Switzerland

E-mail: yuri@math.unibas.ch
Algorithmique Arithmétique Expérimentale (A2X)
UMR CNRS 9936

Université Bordeaux 1

351, cours de la Libération

F-33405 Talence Cedex, France

Current address:

Projet POLKA, INRIA Lorraine

Technopole de Nancy-Brabois

615, rue du Jardin Botanique

B.P. 101

F-54600 Villers-les-Nancy, France

E-mail: Guillaume.Hanrot@loria.fr

Received on 11.4.1997

and in revised form on 4.1.1999 